\title{
Does electronic coherence enhance anticorrelated pigment vibrations under realistic conditions?
}

Cite as: J. Chem. Phys. 151, 114115 (2019); https://doi.org/10.1063/1.5119248

Submitted: 11 July 2019 . Accepted: 30 August 2019 . Published Online: 20 September 2019

Hong-Guang Duan (D), Michael Thorwart (D), and R. J. Dwayne Miller (D)

\section{COLLECTIONS}

Paper published as part of the special topic on Ultrafast molecular sciences by femtosecond photons and electrons

Note: The paper is part of the JCP Special Topic on Ultrafast Molecular Sciences by Femtosecond Photons and Electrons.
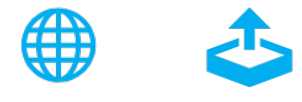

\section{ARTICLES YOU MAY BE INTERESTED IN}

On the calculation of quantum mechanical electron transfer rates

The Journal of Chemical Physics 151, 114119 (2019); https://doi.org/10.1063/1.5116800

X-ray linear and non-linear spectroscopy of the ESCA molecule

The Journal of Chemical Physics 151, 114110 (2019); https://doi.org/10.1063/1.5116699

Ultrafast photoelectron spectroscopy of aqueous solutions

The Journal of Chemical Physics 151, 090901 (2019); https://doi.org/10.1063/1.5098402

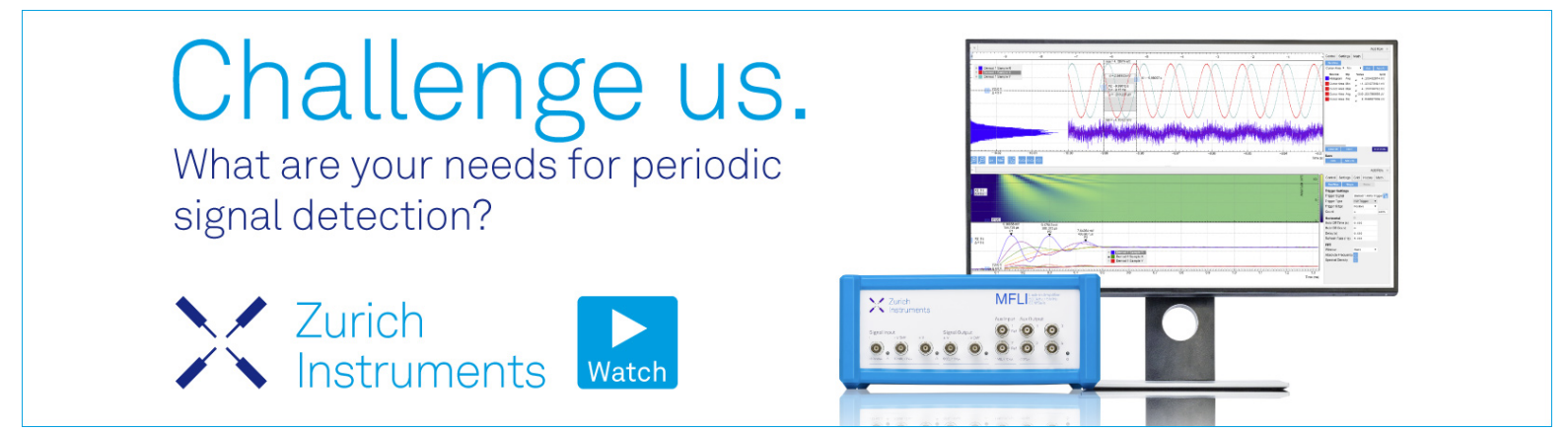




\title{
Does electronic coherence enhance anticorrelated pigment vibrations under realistic conditions?
}

\author{
Cite as: J. Chem. Phys. 151, 114115 (2019); doi: 10.1063/1.5119248 \\ Submitted: 11 July 2019 Accepted: 30 August 2019 • \\ Published Online: 20 September 2019
}

Hong-Guang Duan, ${ }^{1,2,3}$ (D) Michael Thorwart, ${ }^{2,3, a)}$ (D) and R. J. Dwayne Miller ${ }^{1,3,4, b)}$ (D)

\begin{abstract}
AFFILIATIONS
${ }^{1}$ Max Planck Institute for the Structure and Dynamics of Matter, Luruper Chaussee 149, 22761 Hamburg, Germany

${ }^{2}$ I. Institut für Theoretische Physik, Universität Hamburg, Jungiusstraße 9, 20355 Hamburg, Germany

${ }^{3}$ The Hamburg Center for Ultrafast Imaging, Luruper Chaussee 149, 22761 Hamburg, Germany

${ }^{4}$ The Departments of Chemistry and Physics, University of Toronto, 80 St. George Street, Toronto, Ontario M5S 3H6, Canada

Note: The paper is part of the JCP Special Topic on Ultrafast Molecular Sciences by Femtosecond Photons and Electrons.

a) Electronic mail: michael.thorwart@physik.uni-hamburg.de

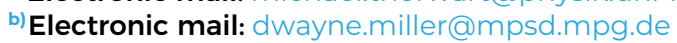

\begin{abstract}
The light-harvesting efficiency of a photoactive molecular complex is largely determined by the properties of its electronic quantum states. Those, in turn, are influenced by molecular vibrational states of the nuclear degrees of freedom. Here, we reexamine two recently formulated concepts that a coherent vibronic coupling between molecular states would either extend the electronic coherence lifetime or enhance the amplitude of the anticorrelated vibrational mode at longer times. For this, we study a vibronically coupled dimer and calculate the nonlinear two-dimensional (2D) electronic spectra that directly reveal electronic coherence. The time scale of electronic coherence is initially extracted by measuring the antidiagonal bandwidth of the central peak in the $2 \mathrm{D}$ spectrum at zero waiting time. Based on the residual analysis, we identify small-amplitude long-lived oscillations in the cross-peaks, which, however, are solely due to groundstate vibrational coherence, regardless of having resonant or off-resonant conditions. Our studies neither show an enhancement of the electronic quantum coherence nor an enhancement of the anticorrelated vibrational mode by the vibronic coupling under ambient conditions.
\end{abstract}

Published under license by AIP Publishing. https://doi.org/10.1063/1.5119248

\section{INTRODUCTION}

In the initial steps of photosynthesis, photoactive molecular complexes capture the sunlight energy and transfer it to the reaction center on an ultrafast time scale with unity quantum efficiency. ${ }^{1}$ The performance is determined by the molecular electronic properties, in concert with the molecular vibrations and coupling to the environment given by a solvent and the surrounding pigments and proteins. To investigate the energy transfer, ultrafast $2 \mathrm{D}$ electronic spectroscopy ${ }^{2-4}$ is able to resolve femtosecond time scales. It is able to reveal the interactions between the energetically close-by lying molecular electronic states, for which the linear spectra are commonly highly congested and broadened by the strong static disorder. ${ }^{5}$ Recent experimental studies of the
Fenna-Matthews-Olson (FMO) complex reported long-lived oscillations of the cross-peaks both at low ${ }^{6}$ and at room temperatures, which have been assigned to enhanced electronic coherence. This has generated tremendous interest in this new field of quantum biology, ${ }^{8}$ aiming to reveal a functional connection between photosynthetic energy transfer and long-lived quantum coherence. Moreover, also in photoactive marine cryptophyte algae, ${ }^{9}$ the light-harvesting complex LHCII, ${ }^{10}$ and the Photosystem II reaction center, ${ }^{11,12}$ longlived oscillations have been experimentally reported at low and room temperatures.

To model the reported ${ }^{6}$ coherence, Ishizaki and Fleming have used a parametrized model of the FMO complex, ${ }^{13}$ with a rather small reorganization energy of $35 \mathrm{~cm}^{-1}$ to fit the electronic coherence time scale. ${ }^{14}$ This value was extracted ${ }^{15}$ from fluorescence 
line narrowing measurements at low temperature ${ }^{16}$ and does not include high-frequency intramolecular modes. However, even with the small reorganization energy, Shi et al. have calculated the complete 2D spectra and found a much shorter electronic coherence lifetime. ${ }^{17}$ They have pointed out that the interpretation of the long-lived coherence could just be due to the intentional magnification of the 2D spectral amplitudes by the deliberately used inverse hyperbolic sine scale. In addition, electronic quantum coherence has been questioned to play any crucial role for the energy transfer as the transport is dominated largely by incoherent exciton relaxation. ${ }^{18,19}$ A critical issue has been the use of an inadequate spectral distribution of the environmental fluctuations. The experimentally determined spectral density with a larger reorganization energy ${ }^{15}$ has been used to calculate the dynamics by the quasiadiabatic propagator path integral. ${ }^{18}$ There, a local vibrational mode at $180 \mathrm{~cm}^{-1}$ with a broadening of $29 \mathrm{~cm}^{-1}$ has been included with a total reorganization energy of $100 \mathrm{~cm}^{-1}$. The numerically exact results also show a significantly shorter electronic coherence lifetime. Recent QM/MM-simulations ${ }^{20-24}$ yield site-resolved spectral densities with reorganization energies of $150-200 \mathrm{~cm}^{-1}$. Moreover, it has been shown in a dimer model that after a short decoherence time, the system relaxes to the polaron state due to strong electronic dephasing, which introduces static coherence in the exciton basis. ${ }^{25}$ This static coherence (or spatial delocalization) of the excitons, which originates from the systembath entanglement, is persistent and can be relevant for energy transfer.

Thus, theoretical studies showed that pure electronic quantum coherence cannot survive under ambient conditions. Motivated by this disagreement, the coherent exciton dynamics in the FMO complex has been reexamined experimentally by $2 \mathrm{D}$ electronic spectroscopy. ${ }^{26}$ A fit to an Ohmic spectral density with a broadened effective high-frequency mode yields a reorganization energy of $190 \mathrm{~cm}^{-1}$. The observed lifetime of the electronic coherence of $\sim 60 \mathrm{fs}$ is too short to play any functional role in the energy transport, which occurs of the picosecond time scale.

In addition to the electronic coherence, signatures of the vibrational coherence of the pigment-protein host can also be accessed on the same spectroscopic footing. ${ }^{27-30}$ Yet, electronic coherence can be distinguished from vibrational coherence. ${ }^{31,34,35}$ Long-lived pure electronic coherence is unexpected to exist in most light-harvesting complexes. However, long-lived vibrational coherence is common and is not expected to strongly affect light-harvesting in the first place. Two concepts are currently under debate: (i) In 2013, Plenio et $a l^{32}$ argued that long-lived vibrationally coherent modes can significantly enhance the electronic coherence lifetime when the vibrational and electronic degrees of freedom are resonantly coupled. The vibrational mode is supposed to act as a "phonon laser" on the excitons, thereby producing ultralong electronic coherence. (ii) Moreover, Tiwari et al. ${ }^{33}$ argued that nonadiabatic electronicvibrational coherent mixing at short times resonantly enhances the amplitude of the particular delocalized anticorrelated vibrational mode in the ground electronic state. This second concept does not involve long-lived electronic coherence and is conceptually in agreement with the observation that a strong vibronic coupling produces large-amplitude coherent oscillations of the electronic component with a usual short lifetime and a long-lived vibrational coherence, but with a rather small amplitude. ${ }^{34,35}$ This scenario was reexamined again recently ${ }^{36}$ with an explicit coupling to an electronic and a vibrational bath. The result that an increased vibronic coupling survives weak electronic dephasing at short times and induces a resonantly enhanced long-lived vibrational coherence of the anticorrelated mode was presented as an explanation of the longlived coherence signals observed in Ref. 6. However, again, an unrealistically weak electronic damping with a reorganization energy of $35 \mathrm{~cm}^{-1}$ has been used.

Motivated by this discrepancy, we have reexamined the coherent dynamics in a model of a vibronically coupled excitonic dimer with anticorrelated pigment vibrations being a minimal model to reveal the principal mechanism at work. We calculate the $2 \mathrm{D}$ electronic spectra of the model dimer with a typical energy gap as being part of the FMO complex. We use the environmental parameters obtained from the recent FMO experiment. ${ }^{26}$ We examine the electronic coherence lifetime by the antidiagonal bandwidth of the diagonal peak at zero waiting time. The oscillations in the residuals obtained from the global fitting analysis confirm that the long-lived coherence is purely vibrational in nature, irrespective of resonant or nonresonant conditions. To distinguish the coherent dynamics of the electronic excited state, we have calculated the dynamics of the electronic wave packet on the vibrational potential energy surfaces (PESs), accompanied by the projection onto the reaction coordinates. Also here, the vibrational coherence is clearly identified by the oscillations close to the potential minimum. The projection shows that the long-lived oscillations are solely of vibrational origin, which confirms the 2D spectroscopic calculations. Moreover, we show that under realistically strong electronic damping, coherent vibronic coupling at short times does not enhance the amplitude of the anticorrelated vibrational mode, while we recover the mechanism of vibronic enhancement only for unrealistically weak electronic damping.

\section{THEORY}

The model is described by a total Hamiltonian consisting of the system, bath, and system-bath interaction terms, $H=H_{S}+H_{S B}$. The system is a dimer consisting of monomers $A$ and $B$ with site energies $E_{A / B}$, both having the same electronic ground state $|g\rangle$ and the respective electronic single excited states $|A\rangle$ and $|B\rangle$. The double excited state is denoted as $|A B\rangle$. Each electronic excited state couples to a vibrational mode (each to its own mode). The two couplings are such that an anticorrelated out-of-phase oscillation of the two electronic states occurs. In the exciton site basis, we have

$$
\begin{aligned}
H_{S}= & |g\rangle h_{g}\langle g|+| A\rangle h_{A}\langle A|+| B\rangle h_{B}\langle B| \\
& +(|A\rangle V\langle B|+\text { h.c. })+|A B\rangle\left(h_{A}+h_{B}\right)\langle A B| .
\end{aligned}
$$

Here, $h_{g}=\frac{1}{2} \Omega\left(P_{A / B}^{2}+Q_{A / B}^{2}\right), h_{A}=E_{A}+h_{g}-\kappa Q_{A}$, and $h_{B}=E_{B}+h_{g}$ $+\kappa Q_{B}$, respectively. $P_{A / B}$ and $Q_{A / B}$ are the momenta and the coordinates of the two vibrational modes coupled to monomers $A$ and B. We express the vibronic coupling strength between the ground and excited states as $\kappa=\frac{\Omega \Delta}{\sqrt{2}}$, where $\Delta$ is the dimensionless shift of the excited state relative to its ground state. $\Omega$ is the vibrational frequency (both modes are taken with equal characteristics). $V$ denotes the electronic coupling between two electronic excited states $|A\rangle$ and $|B\rangle$. 
For the discussion of the anticorrelated vibrations, it is useful to define new coordinates and momenta according to $Q_{ \pm}=\frac{1}{\sqrt{2}}\left(Q_{A} \pm Q_{B}\right)$ and $P_{ \pm}=\frac{1}{\sqrt{2}}\left(P_{A} \pm P_{B}\right) .{ }^{37}$ Then, the system Hamiltonian can be written as

$$
\begin{aligned}
H_{S}= & \sum_{n=0,1,2} H_{S, n}=\sum_{n=0,1,2}\left(H_{S, n}^{+}+H_{S, n}^{-}\right), \\
H_{S, 0}^{-}= & \mathbf{1}_{0} h^{-}, \\
H_{S, 1}^{-}= & \mathbf{1}_{1} h^{-}+\left(E_{B}-\Omega Q-\Delta / \sqrt{2}\right)|B\rangle\langle B|+\left(E_{A}+\Omega Q-\Delta / \sqrt{2}\right) \\
& \times|A\rangle\langle A|+V(|A\rangle\langle B|+| B\rangle\langle A|), \\
H_{S, 2}^{-}= & \mathbf{1}_{2}\left(h^{-}+E_{A}+E_{B}\right),
\end{aligned}
$$

with $\left[H_{S, n}^{+}, H_{S, n}^{-}\right]=0$ and $H_{S, n}^{+}=\mathbf{1}_{n}\left(h^{+}-n \Omega Q_{+} \Delta / \sqrt{2}\right)$. The two rotated vibrational modes are given by $h^{ \pm}=\frac{1}{2} \Omega\left(P_{ \pm}^{2}+Q_{ \pm}^{2}\right)$, and the projection operators are $\mathbf{1}=\sum_{n=0,1,2} \mathbf{1}_{n}$ with $\mathbf{1}_{0}=|g\rangle\left\langle g\left|, \mathbf{1}_{1}=\right| A\right\rangle\langle A|$ $+|B\rangle\langle B|$, and $\mathbf{1}_{2}=|A B\rangle\langle A B|$. The transformation of the Hamiltonian is illustrated in Fig. 1.

We choose the same parameters as in Ref. 33 for the system Hamiltonian. This dimer mimics one exciton pair of the FMO complex. The bath part will be discussed below (in particular, we do not choose the same parameters of too weak damping but use our own parameters of Ref. 26). The electronic energy gap is set to $E_{A}-E_{B}$ $=150 \mathrm{~cm}^{-1}$, and the electronic coupling is $V=66 \mathrm{~cm}^{-1}$. Moreover, the dimensionless vibrational shift is set to $\Delta=0.2236$. The pure electronic energy gap without coupling can then be calculated to be $\Delta E$ $=200 \mathrm{~cm}^{-1}$. As in Ref. 33, we model inhomogeneous broadening by a static Gaussian disorder of width $\delta E=26 \mathrm{~cm}^{-1}$. For the vibrational mode, we choose the frequency $\Omega=200 \mathrm{~cm}^{-1}$ that corresponds to the resonant case when the vibronic coupling vanishes (the slight shift of this resonance due to the vibronic coupling does not alter the overall result since we find essentially the same conclusion also for the off-resonant case; see below).

The environment and coupling part $H_{S B}=H_{S B}^{\mathrm{vib}}+H_{S B}^{\mathrm{el}}$ consist of two parts, the vibrational baths that damp the vibrational motions and the electronic bath that generates electronic dephasing and damping. In general, we assume Gaussian fluctuations described in terms of the standard model of dissipative quantum systems. The electronic environment is generated by fluctuating charges in the protein and the solvent and consists of two harmonic oscillator baths, each of which couples to the electronic excited states of monomers A and B, respectively. Thus, we have the Hamiltonian $H_{S B}^{\mathrm{el}}=\sum_{\alpha=A, B} H_{S B, \alpha}^{\mathrm{el}}$ with

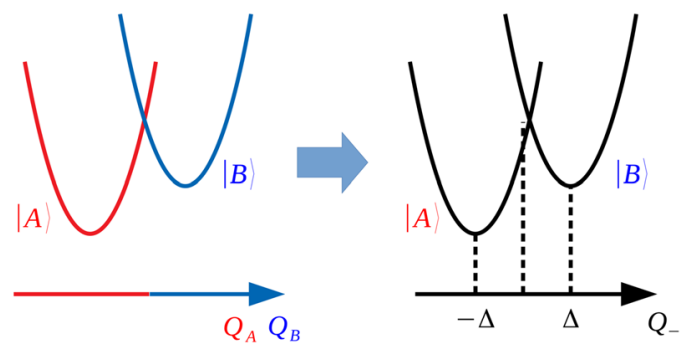

FIG. 1. The vibronic dimer model in Eq. (1) can be transformed into Eq. (2).

$$
H_{S B, \alpha}^{\mathrm{el}}=\frac{1}{2} \sum_{i=1}^{N}\left[\frac{p_{i, \alpha}^{2}}{m_{i, \alpha}}+m_{i, \alpha} \omega_{i, \alpha}^{2}\left(x_{i, \alpha}-\frac{c_{i, \alpha}|\alpha\rangle\langle\alpha|}{m_{i, \alpha} \omega_{i, \alpha}^{2}}\right)^{2}\right] .
$$

As usual, $p_{i, \alpha}$ and $x_{i, \alpha}$ are the momenta and the coordinates of the $i$ th bath mode coupling to the electronic state $\alpha=A, B$. For the electronic part, we choose an Ohmic spectral density with the parameters obtained from fitting the linear spectra of the FMO complex to experimental data (see Ref. 26). Note that these values correspond to much stronger damping than those in Ref. 33. Thus, each bath is assumed to have its own but equal spectral density $J^{\mathrm{el}}(\omega)$ $=\gamma^{\mathrm{el}} \omega \exp \left(-\omega / \omega_{c}\right)$ with $\gamma^{\mathrm{el}}=0.7, \omega_{c}=350 \mathrm{~cm}^{-1}$.

The vibrational environment roots in fluctuating nuclear degrees of freedom of the protein and couples to the vibrational displacements $Q_{A}$ or $Q_{B}$ of the mode coupled to the electronic state $A$ or $B$. Hence, $H_{S B}^{\mathrm{vib}}=\sum_{\alpha=A, B} H_{S B, \alpha}^{\mathrm{vib}}$ with

$$
H_{S B, \alpha}^{\mathrm{vib}}=\frac{1}{2} \sum_{i=1}^{N}\left[\frac{q_{i, \alpha}^{2}}{\mu_{i, \alpha}}+\mu_{i, \alpha} v_{i, \alpha}^{2}\left(y_{i, \alpha}-\frac{d_{i, \alpha} Q_{\alpha}}{\mu_{i, \alpha} v_{i, \alpha}^{2}}\right)^{2}\right] .
$$

$q_{i, \alpha}$ and $y_{i, \alpha}$ are the momenta and the coordinate of the $i$ th vibrational bath mode of the state $\alpha=A, B$. We assume that the vibrational bath has the same spectral density as the electronic bath, i.e., $J^{\text {vib }}(\omega)$ $=\gamma^{\text {vib }} \omega \exp \left(-\omega / \omega_{c}\right)$, but with weaker damping, $\gamma^{\text {vib }}=0.02$ and $\omega_{c}$ $=350 \mathrm{~cm}^{-1}$.

To disentangle electronic and vibrational coherence, we perform a projection of the electronic wave packet on the reaction coordinate, which allows us to distinguish the vibrational coherence from the vibronic dynamics. We assume the initial wave packet to be in the lowest vibrational state $|0\rangle$ of the electronic excited state $|A\rangle$ in the site basis such that the initial density matrix can be written as $\rho(0)=|A, 0\rangle\langle A, 0|$. In order to obtain dynamical information, we determine the probability of the wave packet along the reaction coordinate $Q_{-}$by the time-dependent projection

$$
P_{k}^{a d}\left(Q_{-}, t\right)=\left\langle Q_{-}|\widetilde{k}| \rho(t) \mid \widetilde{k}\right\rangle\left|Q_{-}\right\rangle,
$$

where $P_{k}^{a d}$ is the probability density of the reaction coordinate and $\widetilde{k}$ indicates the electronic state of A or B in the exciton basis (for details of the projection, see Refs. 38 and 39).

\section{RESULTS AND DISCUSSION}

We assume ${ }^{33}$ that two perpendicular transitions from the common ground state to the two excited states of monomers A and $B$ are possible. Hence, the transition dipole moments are fixed to $\vec{\mu}_{A}=\mu_{A} \mathbf{e}_{x}$ and $\vec{\mu}_{B}=\mu_{B} \mathbf{e}_{y}$ with $\mu_{A}=\mu_{B}=1$. Here, $\mathbf{e}_{j}$ is the unit vectors in the direction $j$. Temperature is set to $300 \mathrm{~K}$, if not stated otherwise. We use the time nonlocal quantum master equation ${ }^{40-43}$ with the equation-of-motion phase-matching approach. ${ }^{44}$ Details are given in the supplementary material Appendix of Ref. 26.

We first consider the vibronic dimer with resonant vibrational coupling for which we obtain the $2 \mathrm{D}$ electronic spectrum shown in Fig. 2(a) (real part). At waiting time $T=0 \mathrm{fs}$, the inhomogeneous broadening can be clearly identified because the spectrum is stretched along the diagonal. It disappears within $50 \mathrm{fs}$ (see the time dependent 2D electronic spectra in the supplementary material). The corresponding $2 \mathrm{D}$ spectrum of the purely electronic dimer without vibrational modes is shown in Fig. 2(b). From the 


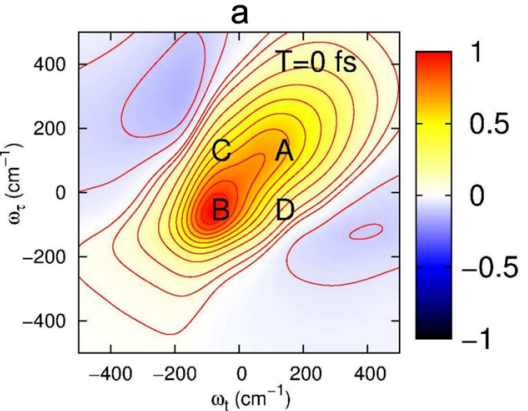

b

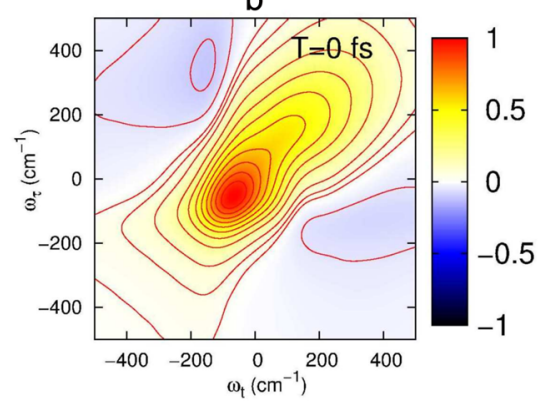

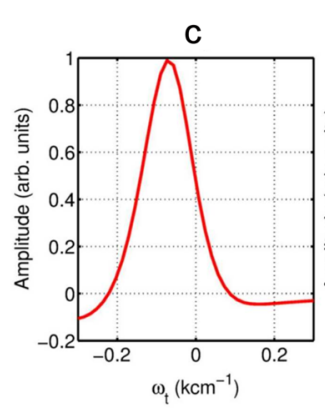
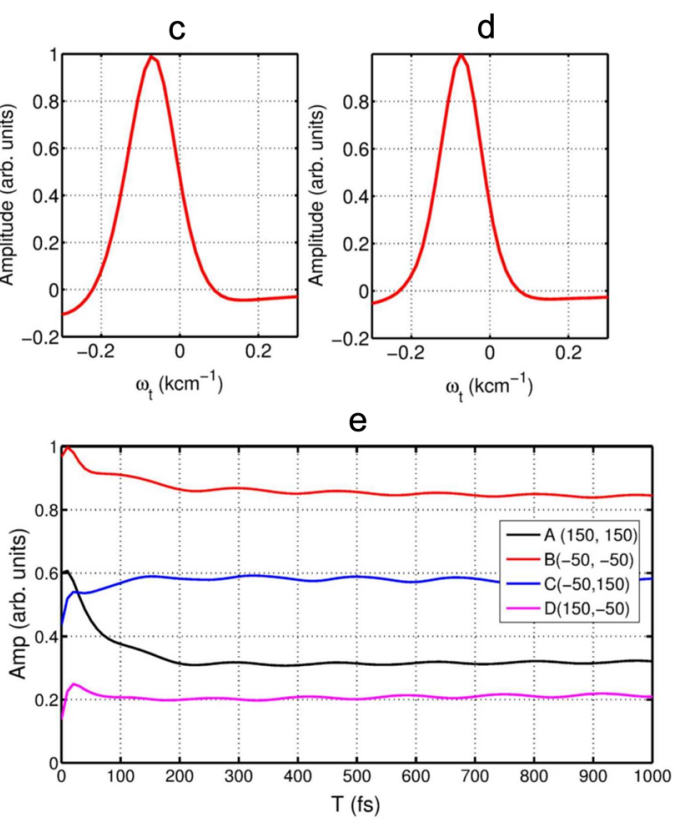

f
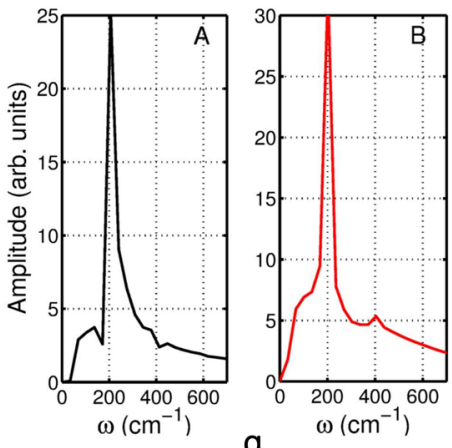

g
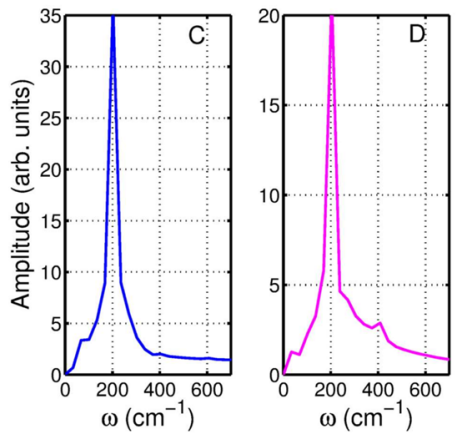

FIG. 2. (a) Real part of the $2 \mathrm{D}$ electronic spectrum of the vibronic dimer at room temperature $(300 \mathrm{~K})$. The diagonal peak $A$ is located at $\left(\omega_{t}=150 \mathrm{~cm}^{-1}, \omega_{\tau}=150 \mathrm{~cm}^{-1}\right)$ and B at $\left(\omega_{t}=-50 \mathrm{~cm}^{-1}, \omega_{\tau}=-50 \mathrm{~cm}^{-1}\right)$. The off-diagonal peak $\mathrm{C}$ sits at $\left(\omega_{t}=-50 \mathrm{~cm}^{-1}, \omega_{\tau}=150 \mathrm{~cm}^{-1}\right)$ and $\mathrm{D}$ at $\left(\omega_{t}=150 \mathrm{~cm}^{-1}, \omega_{\tau}=-50 \mathrm{~cm}^{-1}\right)$. The time dependent trace of the selected peaks is shown in (e). For comparison, the 2D spectrum of the dimer without the effective vibrational mode is shown in (b). To obtain the time scale of the electronic dephasing, the antidiagonal profile of the peaks B in (a) and (b) are shown in (c) and (d). The resulting time scale of the electronic dephasing is 70 fs and $80 \mathrm{fs}$, respectively. [(f) and (g)] Power spectra of the peaks A, B, C, and D for the case with vibrational coupling. The broad background spectral band is associated with fast electronic dephasing. In addition to the strong vibrational peak at $200 \mathrm{~cm}^{-1}$, one additional peak at $400 \mathrm{~cm}^{-1}$ is well resolved.

profiles taken along the antidiagonal band and shown in Figs. 2(c) and $2(\mathrm{~d})$, we observe that the antidiagonal broadening of the $2 \mathrm{D}$ spectra in both cases is similar, which proves that both dimers undergo a similar dephasing dynamics with similar time scales of the electronic dephasing. They are extracted to be $70 \mathrm{fs}$ and $80 \mathrm{fs}$, respectively. To resolve the time-dependent energy transfer, we analyze the series of $2 \mathrm{D}$ spectra for increasing waiting times by the global fitting approach (see the supplementary material for details). This yields the shortest lifetime of the decay associated spectra, which is induced by the peak broadening and by electronic dephasing. Both lifetimes coincide in both cases, which shows that a vibronic coupling does not alter the short-time electronic dephasing properties.

For a quantitative analysis of the dissipative dynamics in the presence of a vibrational coupling, we plot in Fig. 2(e) the time evolution of the magnitude of the peaks selected in Fig. 2(a). We observe that the dynamics can be clearly separated into two sectors: (i) fast electronic dephasing, which initially occurs on the time scale of $\sim 70 \mathrm{fs}$, as already resolved by the analysis of the antidiagonal bandwidth and the global fitting approach. Moreover, (ii) long-lived oscillations with small amplitudes are resolved at longer times. In order to identify the origin of these long-lived oscillations, we perform a Fourier transform of the residual, which is obtained by subtracting the kinetics resolved by the global fitting. The result is shown in Figs. 2(f) and 2(g). The process of fast electronic dephasing is associated with the broad background spectral band with a maximum at $200 \mathrm{~cm}^{-1}$. In addition, this broad band is overlapped by one sharp peak at the same frequency of $200 \mathrm{~cm}^{-1}$. One additional narrow peak is resolved at $400 \mathrm{~cm}^{-1}$. It originates from the vibrational coherence between the vibrational ground $|0\rangle$ and the second vibrational level $|2\rangle$ of the electronic ground state, i.e., it is of pure vibrational origin. This is further illustrated in Fig. S3 of the supplementary material, where the stick spectrum also indicates the clearly separated electronic and vibrational parts of which the eigenstates are composed. Hence, as a matter of fact, we can conclude that these narrow peaks at $200 \mathrm{~cm}^{-1}$ and $400 \mathrm{~cm}^{-1}$ only stem from the vibrational coherence of each monomer.

\section{A. Off-resonance case}

Up to here, we have studied the vibronic dimer for the resonant case $\Delta E=\Omega$. Next, we investigate the off-resonant case as well and choose $\Omega=500 \mathrm{~cm}^{-1}$. The results are shown in Fig. 3(a), 
a

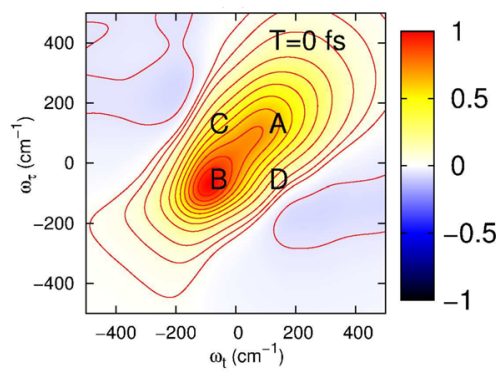

b

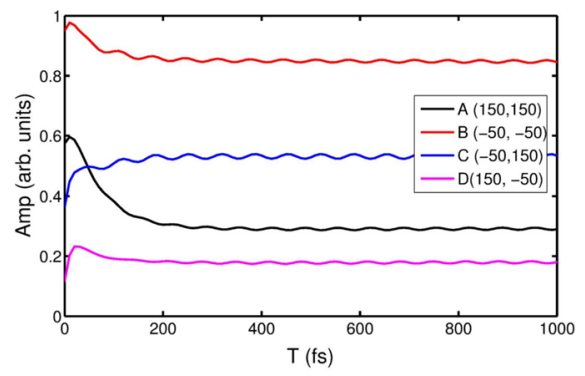

C

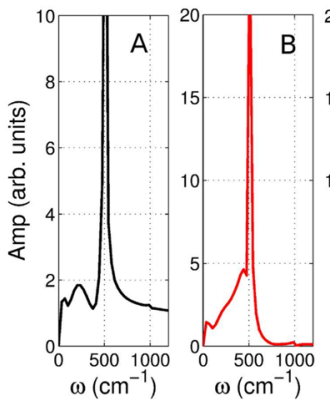

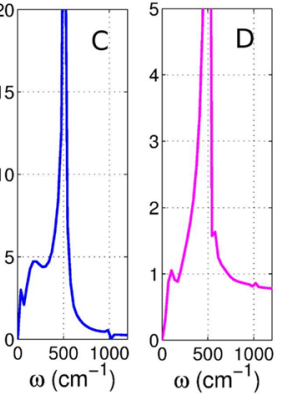

FIG. 3. (a) Real part of the $2 \mathrm{D}$ electronic spectrum of the vibronic dimer under off-resonant conditions with $\Omega=500 \mathrm{~cm}^{-1}$ at $T=0$ fs. The kinetics of the selected peaks at $A$, $\mathrm{B}, \mathrm{C}$, and D are shown in (b), and the associated power spectra are shown in (c). To resolve the lifetime of the electronic coherence, we fit the broad peak at $200 \mathrm{~cm}^{-1}$ with the Lorentzian lineshape and obtain the electronic coherence time of $\sim 70 \mathrm{fs}$, which agrees with the resonant case.

and the global fitting analysis is shown in the supplementary material. The fast electronic dephasing with the time scale of $69 \mathrm{fs}$ is still present. It agrees with the value of the antidiagonal bandwidth (see the supplementary material). Importantly enough, it coincides with the dephasing time scale of the resonant case. Hence, the fact that the electronic and vibrational dynamics are off-resonant does not affect the conclusion reached for the resonant case. In addition, we show in Fig. 3(b) the dynamics of the selected peaks for growing waiting times. It shows the same kinetics as in the resonant case: One fast electronic dephasing component is combined with a long-lived vibrational coherent component with a small amplitude. Again, we perform the Fourier transform of the residuals and plot the spectra of each peak in Fig. 3(c). We again find one broad band with a maximum at $200 \mathrm{~cm}^{-1}$, which manifests the fast electronic dephasing and coincides with the lifetime of $\sim 70$ fs resolved by the global fitting approach. One clearly separated narrow peak is located at $500 \mathrm{~cm}^{-1}$ with a large magnitude which is associated with the long-lived vibrational coherence. A clear evidence for the purely vibrational (and not vibronic) origin of the peak is that one additional peak can be resolved at $\sim 1000 \mathrm{~cm}^{-1}$. It is the clear signature of the vibrational coherence between the vibrational ground $|0\rangle$ and the second vibrationally excited level $|2\rangle$ on the electronic ground-state surface.

\section{B. Low-temperature case}

Next, we consider the case of low temperature of $80 \mathrm{~K}$. We follow the same steps as above and find for vanishing vibronic coupling $\kappa=0$ an electronic dephasing time scale of 161 fs (see Fig. S4 in the supplementary material). In the presence of a resonant vibrational mode with $\Omega=200 \mathrm{~cm}^{-1}$ and with $\kappa \neq 0$ set to the same value as above, we obtain the dephasing time of $121 \mathrm{fs}$ (see Fig. S3(b) in the supplementary material). It is slightly smaller than the one of the purely electronic case, but still comparable. The dynamics of the selected peaks in the 2D spectra again shows long-lived oscillations [see Fig. S3(c) of the supplementary material]. The Fourier transform, shown in Fig. S3(d), shows again one sharp peak at $200 \mathrm{~cm}^{-1}$ and one additional peak at $400 \mathrm{~cm}^{-1}$ with a quite weak magnitude. They manifest again the vibrational origin of the coherence.
Therefore, we can conclude that, also for low temperature, the longlived oscillation is just of vibrational origin. No different mechanism between low and room temperatures occurs.

\section{Vibrational dynamics of the monomer}

In addition to the dimer, we also investigate the monomer where only vibrational coherence is present. In Fig. S5(b) of the supplementary material, we show the time trace of the selected cross peak together with the Fourier spectrum in (c). The spectra are dominated by one peak at the vibrational frequency. An additional peak appears at the position of twice the vibrational frequency. Thus, the same scenario occurs for the monomer as well. We clearly demonstrate that the long-lived oscillations in a vibronically coupled dimer are just due to the overlap of the short-lived electronic coherence and the long-lived vibrational coherence.

\section{Wave packet tracking}

A further confirmation of this picture is obtained from monitoring the dynamics of the electronic excited states. For this, we project the time-evolved density matrix onto the anticorrelated vibrational coordinate $Q_{-}$. We use the same parameters as before and calculate the PESs of the electronic excited states $|\widetilde{A}\rangle$ and $|\widetilde{B}\rangle$ in the adiabatic basis. The result for the off-resonant case with $\Omega=500 \mathrm{~cm}^{-1}$ is shown in Figs. 4(a)-4(d). The initial wave packet is prepared in the excited state $|A\rangle$. For growing time, the transfer of population from $|\widetilde{A}\rangle$ to $|\widetilde{B}\rangle$ can be clearly identified by the decrease of the magnitude in Fig. 4(a) and the corresponding growth in Fig. 4(b). By this, the vibrational coherence of the excited states is clearly visible from the oscillations around the potential minimum [see Fig. 4(a)]. The oscillations have a period of $\sim 66 \mathrm{fs}$, which exactly coincides with the assigned vibrational frequency of $500 \mathrm{~cm}^{-1}$. Moreover, the population dynamics of the states $|\widetilde{A}\rangle$ and $|\widetilde{B}\rangle$ is shown in Fig. 4(c) by summing the wave packet population along the reaction coordinate $Q_{-}$. Spectral information can be again obtained from the Fourier transform. In Fig. 4(d), the vibrational coherence is identified by the narrow peaks at $500 \mathrm{~cm}^{-1}$ and $1000 \mathrm{~cm}^{-1}$, 

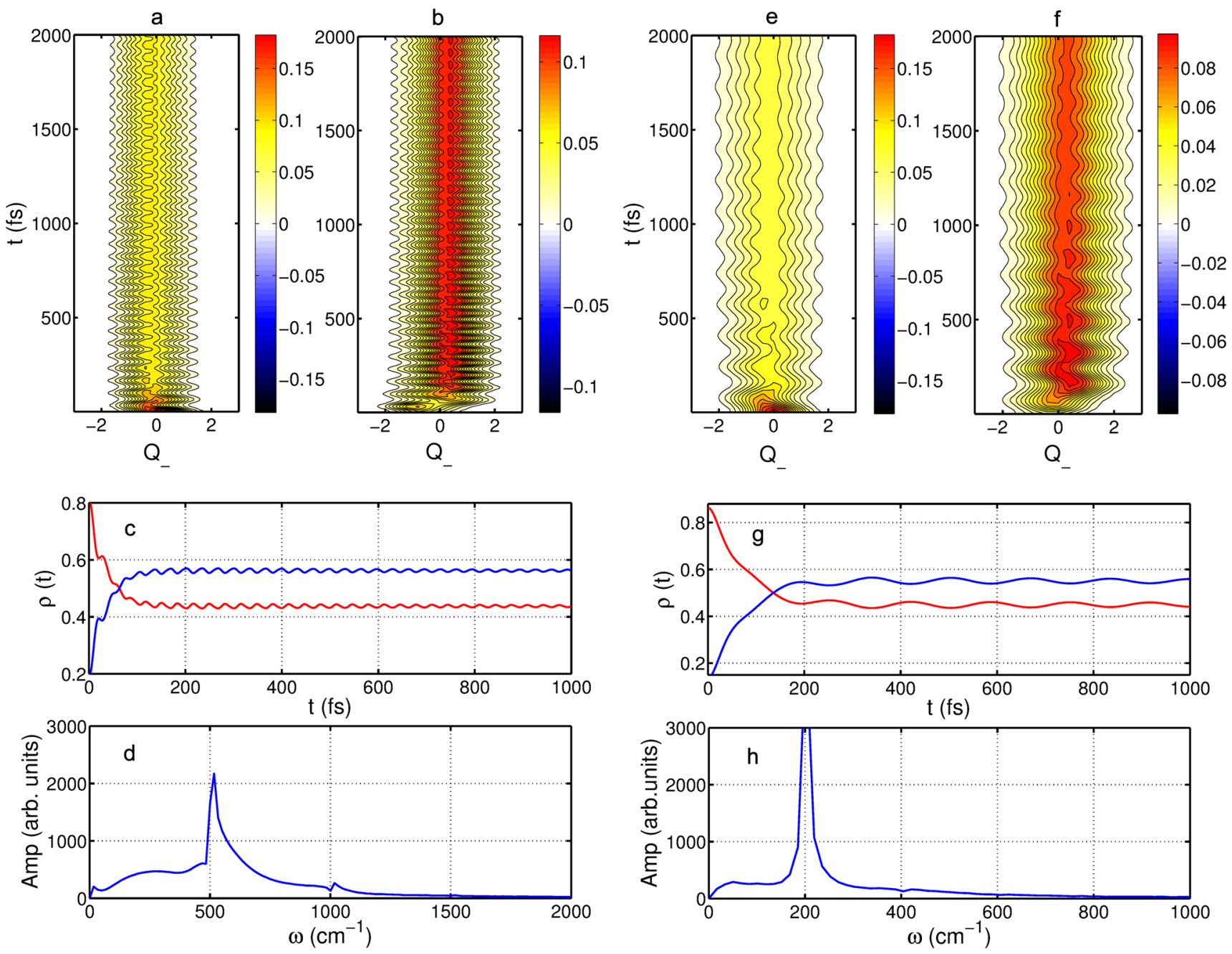

FIG. 4. Time evolution of the wave packet in the excited states $|\widetilde{A}\rangle$ (a) and $|\widetilde{B}\rangle$ (b). The integrated populations of the electronic states $|\widetilde{A}\rangle$ and $|\widetilde{B}\rangle$ obtained by summing along the reaction coordinate $Q_{-}$are shown in (c), together with the Fourier transform of the residuals in (d). The long-lived vibrational coherence is identified by the narrow peaks at $500 \mathrm{~cm}^{-1}$ and $1000 \mathrm{~cm}^{-1}$. In addition, one broadband peak at $200 \mathrm{~cm}^{-1}$ represents the short-lived electronic coherence. The corresponding results of the resonant case are shown in (e)-(h), respectively. The long-lived vibrational coherence can be identified by the narrow peak at $200 \mathrm{~cm}^{-1}$ and the small dip at $400 \mathrm{~cm}^{-1}$.

which coincide with the results from the $2 \mathrm{D}$ spectroscopic calculations shown in Fig. 3. In addition, a broadband background with a maximum at $200 \mathrm{~cm}^{-1}$ and with a small magnitude is visible, which again provides evidence of the electronic coherence being short-lived.

The resonant case, with $\Omega=200 \mathrm{~cm}^{-1}$, is addressed in Figs. 4(e) and 4(f). Compared to the off-resonant case, no significant difference occurs. The initial wave packet in the excited state $|\widetilde{A}\rangle$ is transferred to $|\widetilde{B}\rangle$ over time. The only difference is the vibrational oscillation period of $\sim 165 \mathrm{fs}$. The integrated time-dependent populations are shown in Fig. 4(g), and the associated spectral information is shown in Fig. 4(h). One narrow peak at $200 \mathrm{~cm}^{-1}$ and one additional dip at $400 \mathrm{~cm}^{-1}$ with quite small magnitude occur. Also here, the result agrees with the observation of the $2 \mathrm{D}$ spectroscopic calculations in Fig. 2.

\section{E. Vibronic dimer under weak electronic dephasing}

Up to here, we have studied the realistic parameters of the electronic dephasing and the vibrational damping constants. The possibility remains that for weaker electronic dephasing, the role of a coherent vibronic coupling could be more pronounced. This is not the case that follows from the dynamics of a vibronic dimer in the off-resonant case with $\Omega=500 \mathrm{~cm}^{-1}$ under (unrealistically) weak electronic dephasing. For this, we set $\gamma^{\text {el }}=\gamma^{\text {vib }}=0.02$ and $\omega_{c}=50 \mathrm{~cm}^{-1}$. The wave packet dynamics projected to the PESs 
of $|\widetilde{A}\rangle$ and $|\widetilde{B}\rangle$ is shown in Figs. 5(a) and 5(b), respectively. The purely vibrational coherence can be seen from the wave packet oscillations around $Q_{-}=-1.5$ with a period of $\sim 67 \mathrm{fs}$, which coincides with the vibrational period. The electronic coherence is visible in Fig. 5 as a large-amplitude population exchange between the two electronic states. The electronic oscillation period of $\sim 167 \mathrm{fs}$ corresponds to the electronic energy gap $\Delta E=200 \mathrm{~cm}^{-1}$ in the adiabatic basis. Thus, the large-amplitude exchange is caused by the superposition of the wave packet components on the two PESs. To reveal the oscillation components and their lifetimes, we sum the wave packet components along the reaction coordinate and plot it in Fig. 5(c). The Fourier spectrum is shown in Fig. 5(d). Two large peaks at $200 \mathrm{~cm}^{-1}$ and $500 \mathrm{~cm}^{-1}$ correspond to the oscillations due to electronic and vibrational coherence, respectively. Two small vibronic peaks at $500-200=300 \mathrm{~cm}^{-1}$ and $500+200=700 \mathrm{~cm}^{-1}$ are due to the vibronic mixing. Most importantly, although the frequencies indeed mix and additional peaks are generated, the line widths of the peaks at $200 \mathrm{~cm}^{-1}, 300 \mathrm{~cm}^{-1}, 500 \mathrm{~cm}^{-1}$, and $700 \mathrm{~cm}^{-1}$ are $35 \mathrm{~cm}^{-1}, 40 \mathrm{~cm}^{-1}, 20 \mathrm{~cm}^{-1}$, and $45 \mathrm{~cm}^{-1}$, respectively, and are thus all comparable. This proves that the lifetime of the electronic coherence is not affected by vibronic coupling to a vibrational mode.

\section{F. Impact of coherent vibronic coupling on anticorrelated vibrations}

Finally, we address the possibility that a strong coherent vibronic coupling could enhance the amplitude of the anticorrelated component of the vibrational dynamics. ${ }^{33,36}$ The latter is given by the magnitude of the vibrational peak in the Fourier spectrum of

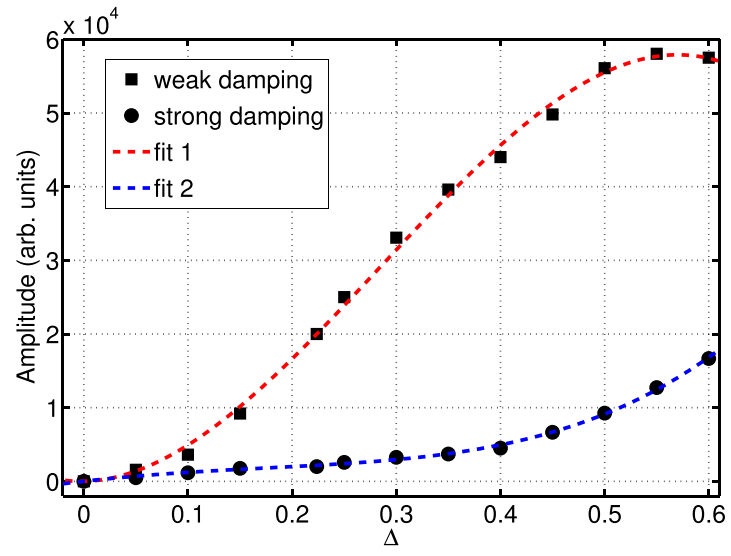

FIG. 6. Oscillation amplitude of the anticorrelated vibration vs the vibronic coupling strength $\Delta$ for weak $\left(\gamma^{\mathrm{el}}=0.02, \omega_{c}=50 \mathrm{~cm}^{-1}\right)$ and strong $\left(\gamma^{\mathrm{el}}=0.7\right.$, $\omega_{c}=350 \mathrm{~cm}^{-1}$ ) electronic dephasing for $\Delta E=200 \mathrm{~cm}^{-1}, \Omega=500 \mathrm{~cm}^{-1}$, and $T=300 \mathrm{~K}$

the wave-packet dynamics, as, e.g., shown in Figs. 4(d), 4(h), and 5(d). In Fig. 6, we show the amplitude of the anticorrelated vibration for increasing vibronic coupling $\Delta$ for weak $\left(\gamma^{\mathrm{el}}=0.02\right)$ and strong $\left(\gamma^{\mathrm{el}}=0.7\right)$ electronic dephasing. For increasing $\Delta$, the mixing of the anticorrelated vibration with the electronic parts becomes stronger. Indeed, for weak electronic dephasing, we find an increase in the anticorrelated vibrational amplitude, which confirms the picture of Refs. 33 and 36. However, for the more realistic case of stronger a

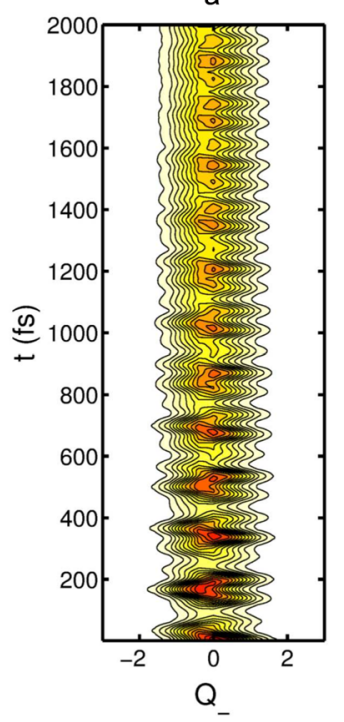

b

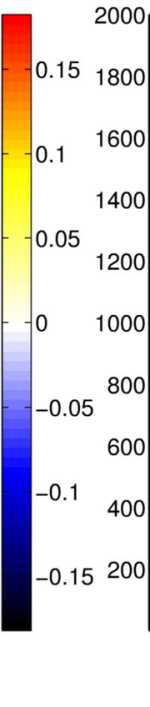

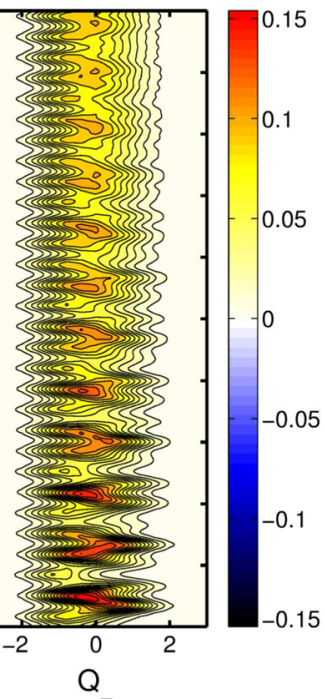

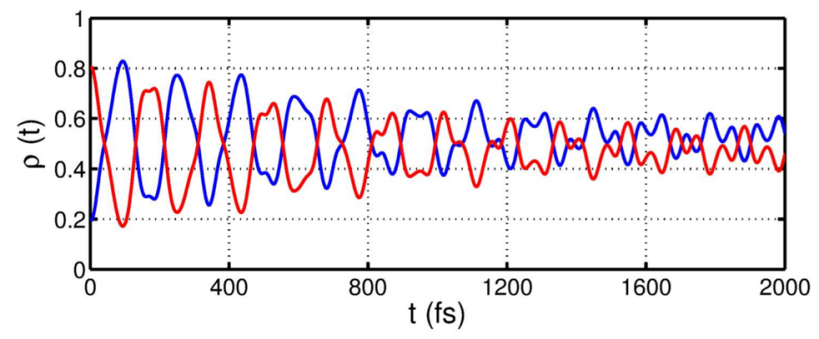

d

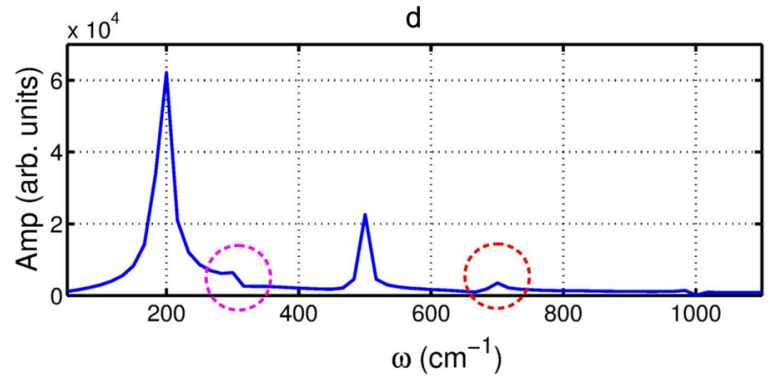

FIG. 5. Time evolution of the wave packet on the excited state PESs for $|\widetilde{A}\rangle$ is shown in (a) and for $|\widetilde{B}\rangle$ in (b) for the vibronic dimer under off-resonant conditions for very weak electronic dephasing $\gamma^{\mathrm{el}}=\gamma^{\text {vib }}=0.02$ and $\omega_{c}=50 \mathrm{~cm}^{-1}$. The integrated populations of the electronic states $|\widetilde{A}\rangle$ and $|\widetilde{B}\rangle$ obtained by summing along the reaction coordinate $Q_{-}$are shown in (c), together with the Fourier transform of the residuals shown in (d). The vibronic coherence can be identified by the two peaks $300 \mathrm{~cm}^{-1}$ and $700 \mathrm{~cm}^{-1}$, which are marked by circles. 
electronic dephasing, the amplitude of the anticorrelated vibration depends only weakly on the vibronic coupling since the coherent electron-vibrational mixing is dephased very rapidly and does not influence the vibration at later times.

\section{CONCLUSIONS}

In conclusion, we have shown in a vibronic dimer model that, under typical ambient physical conditions, it is irrelevant for the lifetime of electronic quantum coherence in the excitation energy transfer whether the two exciton states couple to the two anticorrelated or correlated vibrational modes. This holds irrespective of whether the electronic and vibrational transitions are resonant or off-resonant and follows from an analysis of a model dimer in which each excited state is coupled to its own vibrational mode in an anticorrelated manner. Two independent baths for electronic dephasing as well as vibrational damping are included. By this, we clarify the question in the literature ${ }^{32}$ whether a coupling to long-lived vibrational mode can lead to a substantial increase in the electronic coherence time. The conclusions are drawn from the calculated dynamics, the $2 \mathrm{D}$ electronic spectra, and the subsequent 2D global fitting approach. The exciton dynamics is characterized by the combination of a fast electronic dephasing and a long-lived vibrational coherent component, which has very small oscillation amplitudes. The long-lived oscillations are solely due to the coherence between different vibrational levels, irrespective of a resonant or an off-resonant vibronic anticorrelated coupling. Even under (unrealistically) weak electronic dephasing, the electronic coherence lifetime is not enhanced by the vibronic components. The same conclusion has been drawn from the study of indocarbocyanine dye molecules. ${ }^{34}$ In addition, we find that a strong mixing of electronic and anticorrelated vibrational components of the wavefunction due to strong vibronic coupling does not enhance the vibrational amplitude at long times under ambient conditions. This effect can only occur under unrealistically weak electronic dephasing ${ }^{33,36}$ but is unlikely to play a role in realistic physical systems, the reason being that the required coherent vibronic mixing is rapidly destroyed by fast electronic dephasing.

\section{SUPPLEMENTARY MATERIAL}

See the supplementary material for additional details on the time evolution of $2 \mathrm{D}$ electronic spectra of the vibronically coupled dimer, the global fitting approach, the decay associated spectrum and its form of the vibronically coupled dimer for both the resonant and off-resonant cases, and the comparison to the purely excitonic dimer model without vibrational coupling. Moreover, 2D electronic spectra for $\mathrm{T}=77 \mathrm{~K}$ and of a monomer coupled to a vibrational mode are presented.

\section{ACKNOWLEDGMENTS}

We acknowledge financial support by the Max Planck Society and the Hamburg Centre for Ultrafast Imaging (CUI) within the German Excellence Initiative supported by the Deutsche Forschungsgemeinschaft.

\section{REFERENCES}

${ }^{1}$ R. E. Blankenship, "Antenna complexes and energy transfer processes," in Molecular Mechanisms of Photosynthesis (Blackwell Science, Oxford, Malden, 2002), pp. 61-94.

${ }^{2}$ D. M. Jonas, "Two-dimensional femtosecond spectroscopy," Annu. Rev. Phys. Chem. 54, 425-463 (2003).

${ }^{3}$ M. L. Cowan, J. P. Ogilvie, and R. J. D. Miller, "Two-dimensional spectroscopy using diffractive optics based phased-locked photon echoes," Chem. Phys. Lett. 386, 184-189 (2004).

${ }^{4}$ T. Brixner, T. Mančal, I. V. Stiopkin, and G. R. Fleming, "Phase-stabilized two-dimensional electronic spectroscopy," J. Chem. Phys. 121, 4221-4236 (2004).

${ }^{5}$ S. Mukamel, "Nonlinear response functions and optical susceptibilities," in Principles of Nonlinear Optical Spectroscopy (Oxford University Press, Oxford, 1995), pp. 111-139.

${ }^{6} \mathrm{G}$. S. Engel et al., "Evidence for wavelike energy transfer through quantum coherence in photosynthetic systems," Nature 446, 782-786 (2007).

${ }^{7}$ G. Panitchayangkoona et al., "Long-lived quantum coherence in photosynthetic complexes at physiological temperature," Proc. Natl. Acad. Soc. U. S. A. 107, 12766-12770 (2010).

${ }^{8}$ N. Lambert, Y. N. Chen, Y. C. Cheng, C. M. Li, G. Y. Chen, and F. Nori, "Quantum biology," Nat. Phys. 9, 10-18 (2013).

${ }^{9}$ E. Collini, C. Y. Wong, K. E. Wilk, P. M. G. Curmi, P. Brumer, and G. D. Scholes, "Coherently wired light-harvesting in photosynthetic marine algae at ambient temperature," Nature 463, 644-647 (2010).

${ }^{10} \mathrm{G}$. S. Schlau-Cohen et al., "Elucidation of the timescales and origins of quantum electronic coherence in LHCII," Nat. Chem. 4, 389-395 (2012).

${ }^{11}$ F. D. Fuller, J. Pan, A. Gelzinis, V. Butkus, S. S. Senlik, D. E. Wilcox, C. F. Yocum, L. Valkunas, D. Abramavicius, and J. P. Ogilvie, "Vibronic coherence in oxygenic photosynthesis," Nat. Chem. 6, 706-711 (2014).

${ }^{12}$ E. Romero, R. Augulis, V. I. Novoderezhkin, M. Ferretti, J. Thieme, D. Zigmantas, and R. van Grondelle, "Quantum coherence in photosynthesis for efficient solar-energy conversion," Nat. Phys. 10, 676-682 (2014).

${ }^{13} \mathrm{~A}$. Ishizaki and G. R. Fleming, "Theoretical examination of quantum coherence in a photosynthetic system at physiological temperature," Proc. Natl. Acad. Soc. U. S. A. 106, 17255-17260 (2009).

${ }^{14}$ M. B. Plenio, J. Almeida, and S. F. Huelga, "Origin of long-lived oscillations in 2D-spectra of a quantum vibronic model: Electronic versus vibrational coherence," J. Chem. Phys. 139, 235102 (2013).

${ }^{15} \mathrm{~J}$. Adolphs and T. Renger, "How proteins trigger excitation energy transfer in the FMO complex of green sulfur bacteria," Biophys. J. 91, 2778-2797 (2006).

${ }^{16}$ M. Wendling, T. Pullerits, M. A. Przyjalgowski, S. I. E. Vulto, T. J. Aartsma, R. van Grondelle, and H. van Amerongen, "Electron-vibrational coupling in the Fenna-Matthews-Olson complex of prosthecochloris aestuarii determined by temperature-dependent absorption and fluorescence line-narrowing measurements," J. Phys. Chem. B 104, 5825-5831 (2000).

${ }^{17}$ L. Chen, R. Zheng, Y. Jing, and Q. Shi, "Simulation of the two-dimensional electronic spectra of the Fenna-Matthews-Olson complex using the hierarchical equations of motion method," J. Chem. Phys. 134, 194508 (2011).

${ }^{18} \mathrm{P}$. Nalbach, D. Braun, and M. Thorwart, "Exciton transfer dynamics and quantumness of energy transfer in the Fenna-Matthews-Olson complex," Phys. Rev. E 84, 041926 (2011).

${ }^{19}$ J. Wu, F. Liu, J. Ma, R. J. Silbey, and J. S. Cao, "Efficient energy transfer in lightharvesting systems: Quantum-classical comparison, flux network, and robustness analysis," J. Chem. Phys. 137, 174111 (2012).

${ }^{20} \mathrm{M}$. K. Lee and D. F. Coker, "Modeling electronic-nuclear interactions for excitation energy transfer processes in light-harvesting complexes," J. Phys. Chem. Lett. 7, 3171-3178 (2016).

${ }^{21}$ M. K. Lee, P. Huo, and D. F. Coker, "Semiclassical path integral dynamics: Photosynthetic energy transfer with realistic environment interactions," Annu. Rev. Phys. Chem. 67, 639-668 (2016).

${ }^{22}$ S. A. Oh, D. F. Coker, and D. A. W. Hutchinson, "Optimization of energy transport in the Fenna-Matthews-Olson complex via site-varying pigment-protein interactions," J. Chem. Phys. 150, 085102 (2019). 
${ }^{23}$ S. Chandrasekaran, M. Aghtar, S. Valleau, A. Aspuru-Guzik, and U. Kleinekathöfer, "Influence of force fields and quantum chemistry approach on spectral densities of BChl a in solution and in FMO proteins," J. Phys. Chem. B 119, 9995-10004 (2015).

${ }^{24}$ C. Olbrich, J. Strümpfer, K. Schulten, and U. Kleinekathöfer, "Theory and simulation of the environmental effects on FMO electronic transitions," J. Phys. Chem. Lett. 2, 1771-1776 (2011).

${ }^{25} \mathrm{~J}$. Olšina, A. G. Dijkstra, C. Wang, and J. Cao, "Can natural sunlight induce coherent exciton dynamics?,” e-print arXiv:1408.5385 (2014).

${ }^{26}$ H. G. Duan et al., "Nature does not rely on long-lived electronic quantum coherence for photosynthetic energy transfer," Proc. Natl. Acad. Soc. U. S. A. 114, 8493-8498 (2017).

${ }^{27} \mathrm{D}$. Egorova, "Self-analysis of coherent oscillations in time-resolved optical signals," J. Phys. Chem. A 118, 10259-10267 (2014).

${ }^{28} \mathrm{~V}$. Butkus et al., "Vibrational vs. electronic coherences in 2D spectrum of molecular systems," Chem. Phys. Lett. 545, 40-43 (2012).

${ }^{29} \mathrm{~J}$. Seibt and T. Pullerits, "Beating signals in 2D spectroscopy: Electronic or nuclear coherences? Application to a quantum dot model system," J. Phys. Chem. C 117, 18728-18737 (2013).

${ }^{30} \mathrm{C}$. Kreisbeck and T. Kramer, "Long-lived electronic coherence in dissipative exciton dynamics of light-harvesting complexes," J. Phys. Chem. Lett. 3, 2828-2833 (2012)

${ }^{31}$ C. Kreisbeck, T. Kramer, and A. Aspuru-Guzik, "Disentangling electronic and vibronic coherences in two-dimensional echo spectra," J. Phys. Chem. B 117, 9380-9385 (2013).

${ }^{32} \mathrm{~A}$. W. Chin et al., "The role of non-equilibrium vibrational structures in electronic coherence and recoherence in pigment-protein complexes," Nat. Phys. 9, 113-118 (2013).

${ }^{33}$ V. Tiwari, W. K. Peters, and D. M. Jonas, "Electronic resonance with anticorrelated pigment vibrations drives photosynthetic energy transfer outside the adiabatic framework," Proc. Natl. Acad. Soc. U. S. A. 110, 1203-1208 (2013).

${ }^{34} \mathrm{H}$. G. Duan et al., "On the origin of oscillations in two-dimensional spectra of excitonically-coupled molecular systems," New J. Phys. 17, 072002 (2015).

${ }^{35}$ A. Halpin et al., "Two-dimensional spectroscopy of a molecular dimer unveils the effects of vibronic coupling on exciton coherences," Nat. Chem. 6, 196-201 (2014).

${ }^{36}$ S.-H. Yeh, R. D. Hoehn, M. A. Allodi, G. S. Engel, and S. Kais, "Elucidation of near-resonance vibronic coherence lifetimes by nonadiabatic electronicvibrational state character mixing," Proc. Natl. Acad. Soc. U. S. A. 116, 18263 (2019).

${ }^{37}$ M. F. Gelin et al., "Bath-induced correlations and relaxation of vibronic dimers," J. Chem. Phys. 136, 034507 (2012).

${ }^{38} \mathrm{U}$. Manthe and H. Köppel, "Dynamics on potential energy surfaces with a conical intersection: Adiabatic, intermediate, and diabatic behavior," J. Chem. Phys. 93, 1658-1669 (1990).

${ }^{39}$ D. Qi, H. G. Duan, Z. L. Sun, R. J. D. Miller, and M. Thorwart, "Tracking an electronic wave packet in the vicinity of a conical intersection," J. Chem. Phys. 147, 074101 (2017).

${ }^{40}$ J. S. Cao, "A phase-space study of Bloch-Redfield theory," J. Chem. Phys. 107, 3204-3209 (1997).

${ }^{41}$ C. Meier and D. J. Tannor, "Non-Markovian evolution of the density operator in the presence of strong laser fields," J. Chem. Phys. 111, 3365-3376 (1999).

${ }^{42} \mathrm{U}$. Kleinekathöfer, "Non-Markovian theories based on a decomposition of the spectral density," J. Chem. Phys. 121, 2505-2514 (2004).

${ }^{43}$ Y. C. Cheng and G. R. Fleming, "Coherence quantum beats in two-dimensional electronic spectroscopy,” J. Phys. Chem. A 112, 4254-4260 (2008).

${ }^{44}$ M. F. Gelin, D. Egorova, and W. Domcke, "Efficient method for the calculation of time- and frequency-resolved four-wave mixing signals and its application to photon-echo spectroscopy," J. Chem. Phys. 123, 164112 (2005). 